\section{CLINICAL AND LABORATORY PARAMETERS OF NEONATAL CHOLESTASIS: A \\ RETROSPECTIVE STUDY IN DR. SOETOMO HOSPITAL SURABAYA}

\section{S. Arief}

\section{Pediatric, Dr. Soetomo Hospital/Airlangga University, Surabaya, Indonesia}

Background: Early diagnosis of neonatal cholestasis is important for proper management. Making the differential diagnosis of extrahepatic from intrahepatic cholestasis as the first step for diagnosis is also crucial for better prognosis.

Objective: To find out differences of clinical and laboratory parameters of patients with intrahepatic and extrahepatic neonatal cholestasis.

Method: A retrospective study in infants with neonatal cholestasis who admitted to department of pediatric dr. Soetomo Hospital since 1999 to 2009 was done. Clinical findings (age, gender, birth weight, jaundice, acholic, choluria, hepatomegaly) and laboratory findings (bilirubin, AST, ALT, ALP, GGT) were noted and analyzed with chi-square and independent t-test.

Results: One hundred and nineteen infants with cholestasis were enrolled. Clinical parameters between intrahepatic and extrahepatic neonatal cholestasis included birth-weight $(p=0.34)$, gender $(p=0.33)$, acholic stool $(p=0.07)$, choluria $(p=0.29)$, first presentation of jaundice $(p=0.47)$, and hepatomegaly $(p=0.34)$ were not differed significantly. Laboratory parameters, AST $(p=0.87)$, $\operatorname{ALT}(p=0.35)$, alkaline phosphatase $(p=0.57)$, and Gamma-GT $(p=0.08)$ were not differed significantly. Only Total bilirubin $(p=0.001)$, direct bilirubin $(p=0.004)$ were differed significantly, with higher level in extrahepatic neonatal cholestasis.

Conclusion: Bilirubin level in intrahepatic and extrahepatic neonatal cholestasis were significantly different.

\section{OUTCOME OF CZECH INFANTS WITH BILIARY ATRESIA AFTER 10 YEARS PERIOD (1998- 2009)}

R. Kotalova ${ }^{1}$, K. Blahova ${ }^{1}$, M. Rygl ${ }^{2}$, J. Snajdauf ${ }^{2}$, R. Keil ${ }^{3}$

${ }^{1} D p t$. of Paediatrics, ${ }^{2} D p t$. of Paediatric Surgery, ${ }^{3}$ Dpt. of Internal Medicine, 2nd Faculty of Medicine, Charles University in Prague and Motol University Hospital, Prague, Czech Republic

Introduction: Biliary atresia (BA) is a result of an idiopathic destructive obliterative inflammatory cholangiopathy of both intrahepatic and extrahepatic bile ducts. If untreated, progressive liver cirrhosis leads to death. The prevalence of $\mathrm{BA}$ ranging from 1 in 12 000-18 000 lifebirths. Up to 10-20\% of all caases are associated with other congenital abnormalities.

The aim of the study: Analysis of epidemilogic data and courses of Czech children with BA during last 10 years (1/1998-12/2009).

Patiens: All patiens with suspicion of BA were treated in one centre where endoscopic retrograde cholangiopancreatography (ERCP), Kasai portoenterostomy and adjuvant therapy were provided.

Results: Kasai portoenterostomy was performed in all children $(n=75)$ at the median age of 62 days (20-140 days). Associated anomalies (polysplenia, situs viscerum inversus etc.) were found in $6 / 75$ children (8\%).

The incidence of BA in Czech Republic is 1 in 16000 lifebirths (3-11 children per year). The survival rate of children (born before 12/2009) is $76 \%$ (57/75 pts). $42 / 75$ pts $=56 \%$ (aged 4 months - 11.5 years) achieved good quality of life with their native liver. $15 / 75$ pts $(20 \%)$ are after successful liver transplantation. The mortality rate of pts with BA is $24 \%$ (18/75), 3/18 children dead after liver transplantation.

Conclusion: The incidence of BA in Czech Republic did not differ from its incidence in another European countries. This disorder seems to be the most often indication for liver transplantation. 